\title{
Dark Particles Answer Dark Energy
}

\author{
John L. Haller Jr. \\ Predictive Analytics, CCC Information Services, Chicago, USA \\ Email: jlhaller@gmail.com
}

Received April 11, 2013; revised May 13, 2013; accepted June 15, 2013

Copyright (C) 2013 John L. Haller Jr. This is an open access article distributed under the Creative Commons Attribution License, which permits unrestricted use, distribution, and reproduction in any medium, provided the original work is properly cited.

\begin{abstract}
This paper argues that a hypothetical "dark" particle (a black hole with the reduced Planck mass and arbitrary temperature) gives a simple explanation to the open question of dark energy and has a relic density of only $17 \%$ more than the commonly accepted value. By considering an additional near-horizon boundary of the black hole, set by its quantum length, the black hole can obtain an arbitrary temperature. Black-body radiation is still present and fits as the source of the Universe's missing energy. Support for this hypothesis is offered by showing that a stationary solution to the black hole's length scale is the same if derived from a quantum analysis in continuous time, a quantum analysis in discrete time, or a general relativistic analysis.
\end{abstract}

Keywords: Dark Particle; Dark Energy; Black Hole; Black-Body Radiation; Diffusion; Brownian Motion; Langevin Equation; Dark Matter

\section{Introduction}

Cosmological observations from early in the last century indicate the Universe is expanding. These observations show that the speed at which objects move away from Earth has a strong correlation with their distance, known as Hubble's Law. However, it was not until the end of the last century, when we had observations of Type Ia supernova, that we concluded the Universe is also accelerating [1].

The most popular explanation for these findings is an elusive energy density with an equation of state, $w<$ $-1 / 3$ [2] coined "Dark Energy" making up 73\% [3] of the energy density of the Universe. Many attempts have been made to explain dark energy's origin [4], yet those attempts have ultimately been unsatisfying [2,5]. Many theories are still under review [6,7]; with the most accepted being the Lambda Cold Dark Matter model, $\Lambda \mathrm{CDM}$ where Lambda, the Cosmological Constant, provides a negative pressure $w=-1$, to the Universe $[1,2]$.

By hypothesizing a "dark particle" we begin to answer the question of the missing dark energy density. We will see that a dark particle is a black hole with the reduced Planck mass. At this mass, the original assumption in Hawking's work on black hole radiation [8] breaks down and I show how a quantum boundary, set by the width of the wave packet, is larger than the event horizon. The surface gravity at this new quantum boundary is such that the temperature is arbitrary. Still the distribution of kinetic and potential energy is shown to be that of the black-body.

We now have a source of a black-body energy density that contributes to the energy density of the Universe. One might conclude that dark particles are in thermal equilibrium with the Cosmic Microwave Background; however, I will argue that the coupling mechanism between the dark particles and the background radiation field has been turned off since after the Dark Ages when re-ionization happened. If this is the case and if the dark particles temperature is frozen, they have an equation of state of $w=-1$ and the right relic density to explain dark energy.

It is also shown how the dark particles cool via a stationary diffusive process where the quantum solution to width of the wave packet (the near horizon quantum boundary) is derived. I present this analysis in both continuous and discrete space-time and use a computer model to show that the two give the same solution. A linkage between quantum mechanics and gravity is gained by considering three energy density terms of the dark particle. The resulting length scale, as solved by Friedmann's equation, is identical to solution of its quantum length.

Section 2 argues that a black hole with the reduced Planck mass (a dark particle) has an arbitrary temperature and that its density explains dark energy. Section 3 confirms that a dark particle's energy is still in the form 
of black-body radiation. Section 4 solves the length scale of a dark particle from a continuous quantum derivation and explains the mechanism by which the dark particle cools. Section 5 validates the solution to the length scale derived in Section 4 by modeling the diffusive process in discrete space-time. Section 6 shows that an identical solution to the length scale can also be derived from Friedmann's equation when appropriate densities are considered. Section 7 discusses other similar work and how dark particles might solve other open questions as well, such as dark matter.

\section{Missing Energy}

\subsection{Dual Gaussians}

We begin by setting the context on a particle of mass $m$ in equilibrium with a heat bath at temperature $T$. We assume a particle is in the dual Gaussian ground state.

$$
\begin{gathered}
x \sim p(x) d x=\frac{1}{\sqrt{2 \pi\left(\Delta x_{0}\right)^{2}}} \cdot \mathrm{e}^{-\frac{x^{2}}{2\left(\Delta x_{0}\right)^{2}}} d x \\
p_{x} \sim p\left(p_{x}\right) d p_{x}=\frac{1}{\sqrt{2 \pi\left(\Delta p_{0}\right)^{2}}} \cdot \mathrm{e}^{-\frac{p_{x}^{2}}{2\left(\Delta p_{0}\right)^{2}}} d p_{x}
\end{gathered}
$$

Using the equipartition theorem on the kinetic energy [9], one has

$$
\left(\Delta p_{0}\right)^{2}=m k_{B} T
$$

And using Heisenberg's Uncertainty equation [9],

$$
\left(\Delta x_{0}\right)^{2}=\frac{\hbar^{2}}{4\left(\Delta p_{0}\right)^{2}}=\frac{\hbar^{2}}{4 m k_{B} T}
$$

Making use of the equipartition theorem implies that the particle is coupled to an ensemble of particles or heat bath [10]. The bath in this case is an external radiation field (for example, the Cosmic Microware Background). However, as we will see, the coupling between the dark particles and the heat back can be turned on or off with the presence or absence of neutral hydrogen atoms. We will use the temperature at the time of last coupling and the particle's mass to define the width of the wave function.

\subsection{Black Holes}

We will now apply these lengths to our understanding of black holes, specifically holes with a mass equal to the reduced Planck mass.

$$
m=\sqrt{\frac{\hbar c}{8 \pi G}}
$$

A number of special conditions arise at this value of mass. First, the quantum limit, $d x=\hbar / 2 m c$ is equal to a circle's circumference with the Schwarzschild radius, Figure 1.

$$
d x=\frac{\hbar}{2 m c}=2 \pi R_{S}=4 \pi G m
$$

Indeed this is a small cross-sectional area for the black hole.

Second, the Hawking temperature is equal to the mass of the black hole,

$$
k_{B} T_{\text {Hawking }}=\frac{\hbar c^{3}}{8 \pi G m}=m c^{2}=\left(4.3 \times 10^{-6} \text { grams }\right) c^{2}
$$

Third, it is not clear that the Hawking temperature is valid at this value of the mass. Specifically Hawking stated in his seminal paper from 1975 [8], "Eventually, when the mass of the black hole is reduced to $10^{-5} \mathrm{~g}$, the quasi-stationary approximation will break down. At this point, one cannot continue to use the concept of a classical metric. However, the total mass or energy remaining in the system is very small."

Even more recent derivations of Hawking's work still breakdown at this mass [11]. I will argue that when a black hole has the reduced Planck mass, the Hawking temperature breaks down because a secondary quantum boundary is greater than the Schwarzschild radius and it is this boundary that defines the near horizon's surface gravity. The length of the boundary is such that its surface gravity/temperature is arbitrary. I will also argue that even though "the total energy in the system is very small," it has just the right density (given the history of our Universe) to explain dark energy.

\subsection{Quantum Boundary}

As the event horizon is defined by the quantum limit, $d x$, the outer quantum boundary is defined by the square root of the position's variance $\left(\Delta x_{0}\right)$. If $\Delta x_{0}$ defines the circumference of the boundary (as $d x$ defines the circumference of the event horizon), the radius of the outer boundary will be $R_{\mathrm{QB}}$, see Figure 1 .

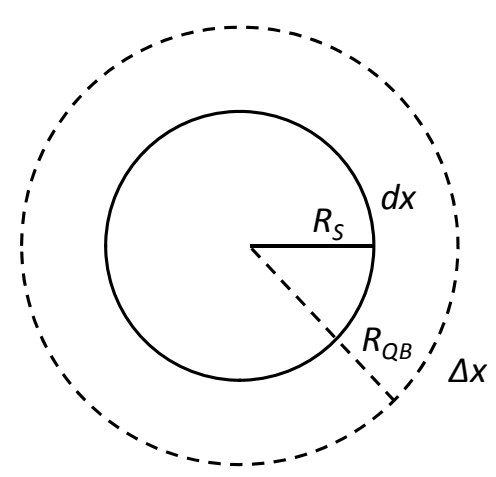

Figure 1. Event horizon (solid line) and quantum boundary (dotted line) of dark particle. 


$$
R_{\mathrm{QB}}=\frac{\Delta x_{0}}{2 \pi}=\frac{\hbar}{4 \pi \sqrt{m k_{B} T}}
$$

The surface gravity at radius $r$ is [12],

$$
\kappa(r)=\frac{-1}{2} \frac{\partial}{\partial r}\left(-1+\frac{2 G m}{c^{2} r}\right)=\frac{G m}{c^{2} r^{2}}
$$

The effective temperature [8] for surface gravity at radius $R_{\mathrm{QB}}$ will thus be,

$$
T_{\text {DarkParticle }}=\frac{\kappa\left(R_{\mathrm{QB}}\right) \hbar}{2 \pi k_{B} c}=\frac{8 \pi G m^{2} T}{\hbar c}=T
$$

The width of the black hole's wave packet (which is set by the temperature of the heat bath) that defines the outer quantum boundary is just the right size to define a surface gravity such that the temperature is arbitrary and not a function of mass or other defining feature of the black hole. The temperature is its own independent parameter of the black hole. Thus I will call a black hole with the reduced Planck mass and arbitrary temperature a dark particle.

When the mass of a black hole is greater than the reduced Planck mass, the quantum boundary $R_{\mathrm{OB}}$ is necessarily smaller than $R_{\text {Schwarzschild }}$ and thus it is $R_{\text {Schwarzschild }}$ that defines the surface gravity. When the black hole is a dark particle it can't lose any more mass lest its quantum limit will become larger than $R_{\text {Schwarzschild; }}$ thus it will cease to be a black hole. If the dark particle loses radiation it must shed its non-massive energy and thus decrease in temperature. On the flip side, if the dark particle is in a heat bath at a higher temperature than the dark particle, it will match that larger temperature (assuming a coupling mechanism is in place) without gaining mass until the reduced Planck temperature is reached.

\subsection{Dark Energy}

Now hypothesize that a local group of dark particles are able to exchange heat with the Cosmic Microwave Background when neutral hydrogen atoms or other sinks are nearby to capture the radiation from its gravitational binding but that they become frozen (constant temperature) when neutral hydrogen atoms are not nearby.

This hypothesis rests on the idea that a virtual particle that leaves its pair becomes trapped by the outer quantum boundary, even if it escapes the event horizon, unless a sink is around to capture it. See Figure 2. With no sink the net energy to escape is zero. However if a sink is around, like a neutral hydrogen atom, the sink can absorb radiation at one energy and release radiation into the dark particle at another energy, keeping it in thermal equilibrium.

During the dark ages, the time between decoupling and re-ionization [13], the Universe was filled with hydrogen
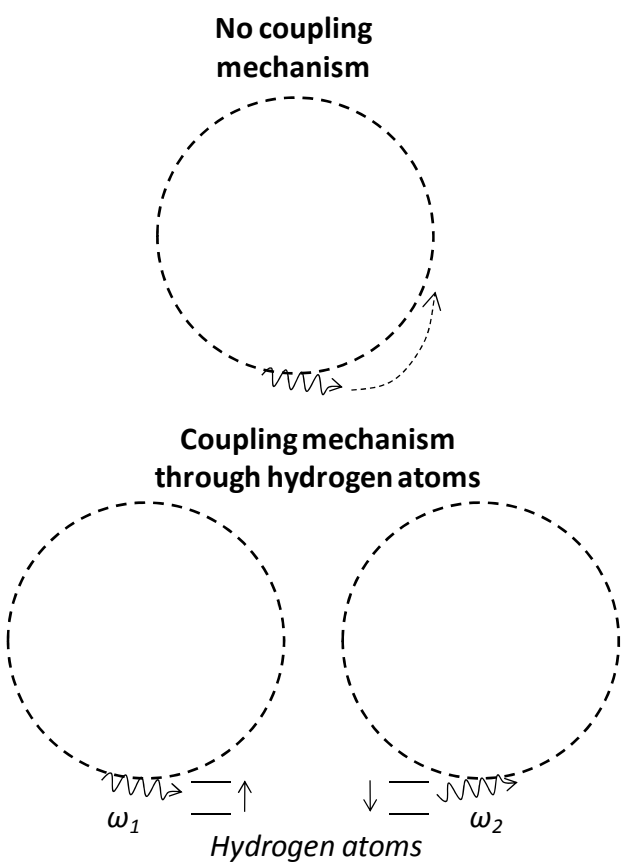

Figure 2. Without a coupling mechanism, the radiation can't escape thereby not allowing the dark particle to cool. However with a hydrogen atom or other sink the dark particle can equilibrate with the background field.

atoms that provided the coupling mechanism between the dark particles and regular energy. In these conditions the dark particles were coupled to the Cosmic Microwave Background (CMB). However after re-ionization, the hydrogen was ionized and the dark particles and its associated radiation energy density became frozen. The red-shift of re-ionization and the current temperature of the CMB provide an estimate of the temperature of the dark particles at the time of re-ionization where it remains constant up to today.

$$
T_{\mathrm{DP}}=\left(1+z_{\text {Re-ionization }}\right) T_{\text {CMB-today }}=\text { constant }
$$

If we know the temperature of the dark particles at re-ionization, then we should have an idea for the total energy density that contributes to the Cosmological constant today.

$$
\rho_{\text {Dark Particles-BBR }}=\frac{\pi^{2}}{15} \frac{\left(k_{B} T_{D P}\right)^{4}}{\hbar^{3} c^{5}}
$$

Because we have estimates of today's $z$ value of reionization and today's temperature of the $\mathrm{CMB}$ we can estimate the density, $\rho_{\text {DP-BBR }}$. The Lambda Cold Dark Matter model, $\Lambda \mathrm{CDM}$, provides a completely independent estimate of the density of dark energy, $\rho_{\Lambda \mathrm{CDM}}$ [1], which we can estimate using the parameter, $\Omega_{\Lambda}$, and today's Hubble constant.

$$
\rho_{\Lambda C D M}=\Omega_{\Lambda} \cdot \rho_{\text {critical }}=\Omega_{\Lambda} \cdot \frac{3 H^{2}}{8 \pi G}
$$


Using the 7-year Wilkinson Microwave Anisotropy Probe [3] as a source for our estimates Table 1 and Figure 3 show how our model's estimate of dark energy is higher by $17 \%$ but well within the confidence range defined by $Z_{\text {re-ionization }}$.

\subsection{Inflation}

It is also supportive to examine how this theory holds up to the inflationary period just after the big bang. The inflationary period that follows the grand unified period lasts for $\sim 10^{-34}$ seconds and during this time, the scale factor of the Universe grows exponentially by a factor of $\sim 10^{26}$ [14]. Assuming that dark particles are able to release heat, thereby maintaining equilibrium with the rest of the Universe's energy during the time of grand unification (immediately preceding inflation), but that once the inflation period began the dark particles became isolated,then the dark particles will have a constant energy density during inflation leading to exponential expansion (but with a much higher rate than today).

The theory also provides insights into reheating, the period after inflation. If you imagine the dark particles were at the temperature of grand unification at the beginning of the inflationary epoch only to become isolated,

Table 1. Estimate and confidence ranges of Dark Energy from the Dark Particles BBR model and the Lambda ColdDark Matter model using 7-year WMAP data.

\begin{tabular}{cccc} 
& Low & Average & High \\
\hline$\rho_{\text {DP-BBR }}$ & $5.22 \mathrm{E}-27$ & $8.12 \mathrm{E}-27$ & $1.21 \mathrm{E}-26$ \\
$\left(\mathrm{~kg} / \mathrm{m}^{3}\right)$ & & & \\
$T_{\text {today }}$ & 2.725 & 2.725 & 2.725 \\
$($ degrees $)$ & 9.3 & 10.5 & 11.7 \\
$Z_{\text {re-ionization }}$ & & & \\
$\rho_{\text {ACDM }}$ & $6.21 \mathrm{E}-27$ & $6.95 \mathrm{E}-27$ & $7.74 \mathrm{E}-27$ \\
$\left(\mathrm{~kg} / \mathrm{m}^{3}\right)$ & & & 73.5 \\
$H$ & 68.5 & 71.0 & 0.763 \\
$(\mathrm{~km} / \mathrm{sec} \cdot \mathrm{Mpc})$ & & & \\
$\Omega$ & 0.705 & 0.734 & \\
\hline
\end{tabular}

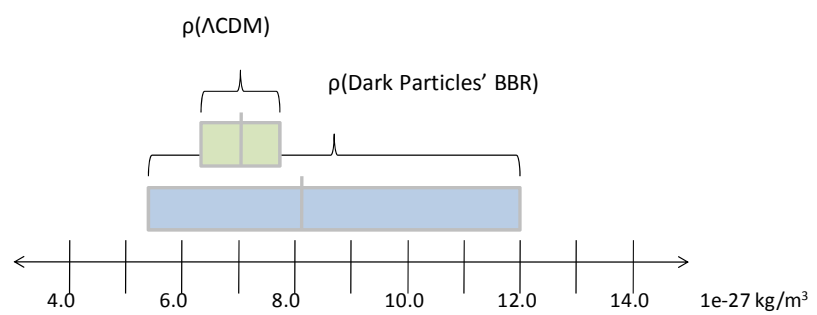

Figure 3. Visualization of density of dark energy from two models. the dark particles would remain constant during the inflation while the rest of the energy density in the Universe would cool by a factor of $\sim\left(10^{26}\right)^{4}$. If quarks, anti-quarks or gluons (which became available at the end of the inflationary period) are able to couple dark particles to the rest of the Universe's energy (as we hypothesized neutral hydrogen atoms are able to do), heat could flow from the hot dark particles back into the rest of the Universe, reheating it.

\section{Black-Body Radiation}

With the derivation of the Hawking temperature breaking down at the reduced Planck mass, the derivation of black-body radiation is also in question. However as we see below, a black hole with the reduced Planck mass and arbitrary temperature still radiates a density of energy with the black-body distribution.

\subsection{Resistive Force}

We begin by assuming aquadratic potential energy term. If the equipartition theorem and Heisenberg Uncertainty principle hold [9] we can derive the potential energy term. We have for one dimension,

$$
P E=C x^{2}
$$

From the equipartition theorem,

$$
\begin{gathered}
\overline{P E}=\overline{K E}=\frac{k_{B} T}{2} \\
C \overline{x^{2}}=\frac{\overline{p^{2}}}{2 m}=\frac{k_{B} T}{2}
\end{gathered}
$$

If $\bar{x}=\bar{p}=0$ and if $\Delta p \Delta x_{0}=\hbar / 2$, one can deduce the potential energy and the associated force

$$
\begin{aligned}
& P E=\frac{m x^{2}}{2 \tau^{2}} \\
& F=\frac{-m x}{\tau^{2}}
\end{aligned}
$$

where,

$$
\tau=\hbar / 2 k_{B} T .
$$

In Section 4 we show how this force is related to kinetic motion. Further in Section 6 we show this resistive force can also be derived from the self-gravitational potential of the particle and thus only acts over the distance defined by the Schwarzschild radius. Since the Schwarzschild radius is much smaller than the quantum step size of any particle we have experimental data on, we have never directly observed this effect. 


\subsection{Energy Density}

Pulling together the kinetic and potential energy terms for all three dimensions we have the energy of the 3-D oscillator

$$
\hbar \omega=\frac{m}{2 \tau^{2}}\left(x^{2}+y^{2}+z^{2}\right)+\frac{1}{2 m}\left(p_{x}^{2}+p_{y}^{2}+p_{y}^{2}\right)
$$

Again as the harmonic oscillator is in the ground state, we see our starting point with dual Gaussian wave functions is justified. Again solutions for position and momentum in the ground state given by Equations (1)-(4).

Thus the particle's wave function is isomorphic to a Wigner function of a point particle at $(x=0, p=0)$ squeezed to assure the equipartition theorem holds for both the kinetic and potential energy [15]. We can solve for the probability distribution on $\omega$ [16].

$$
\omega \sim p(\omega, T) d \omega=\frac{\hbar^{3} \omega^{2}}{2\left(k_{B} T\right)^{3}} \mathrm{e}^{\frac{-\hbar \omega}{k_{B} T}} d \omega
$$

The average energy of this distribution is the 3-D ground state energy of the harmonic oscillator,

$3 \cdot \hbar \omega_{0} / 2=3 / 2 \cdot \hbar \sqrt{\text { spring constant } / m}=3 k_{B} T$. However before we can associate this probability distribution with the internal radiation density, we must account for the fact that multiple photons can occupy the same state [9]. Thus if $M$ is the number of photons we have,

$$
\begin{gathered}
M \cdot \overline{\hbar \omega}=\frac{6 \cdot k_{B} T}{2} \\
M \cdot \overline{\frac{m}{2}\left(\frac{x}{\tau}\right)^{2}}=M \cdot \overline{\frac{1}{2 m}\left(p_{x}\right)^{2}}=\frac{k_{B} T}{2} \\
T \rightarrow \frac{T}{M}
\end{gathered}
$$

And

$$
p_{\text {radiation }}(\omega) d \omega=p\left(\omega, \frac{T}{M}\right) d \omega=\frac{M^{3} \hbar^{3} \omega^{2}}{2\left(k_{B} T\right)^{3}} \mathrm{e}^{\frac{-M \hbar \omega}{k_{B} T}} d \omega
$$

To deduce the radiation density from the probability distribution on the photons, one must divide by the surface area at $c \tau, A=4 \pi(c \tau)^{2}$ and multiply by $1 / c$ times the power which in this case is $\hbar \omega$ divided by one period of the harmonic oscillator, $1 / f_{0}=2 \pi / \omega_{0}=2 \pi \tau$

$$
\begin{aligned}
\rho_{\text {radiation- } M}(\omega) d \omega & =\frac{\hbar \omega}{A \cdot c \cdot 2 \pi \tau} p_{\text {radiation }}(\omega) d \omega \\
& =\frac{\hbar \omega^{3}}{2 \pi^{2} c^{3}} \mathrm{e}^{\frac{-M \hbar \omega}{k_{B} T}} d \omega
\end{aligned}
$$

Lastly, sum over all states of photons $M$ (from 1 to $\infty$ ), and both degrees of polarization [17] since all are possible.

$$
\begin{aligned}
\rho_{\text {radiation }}(\omega) d \omega & =\sum_{\text {pol }=1,-1} \sum_{M=1}^{\infty} \rho_{\text {radiation- }}(\omega) d \omega \\
& =\frac{\hbar \omega^{3} d \omega}{\pi^{2} c^{3}} \sum_{M=1}^{\infty} \mathrm{e}^{\frac{-M \hbar \omega}{k_{B} T}}=\frac{\hbar \omega^{3}}{\pi^{2} c^{3}} \frac{1}{\left(\mathrm{e}^{\frac{\hbar \omega}{k T}}-1\right)} d \omega
\end{aligned}
$$

One will recognize the energy density of Black-body radiation, [17].

\section{Quantum Solution (Continuous Space Time)}

\subsection{Free Particle Diffusion}

We begin with the quantum diffusion of a free particle, which can be derived from the equations of motion [10]. With zero force and $m \dot{x}=p$ one can deduce,

$$
x(t)=x_{0}+\frac{p}{m} t
$$

Calculating the variance and using $\overline{x(\dot{x})}(t=0)=\hbar / 2 m$ we have,

$$
(\Delta x)^{2}(t)=\left(\Delta x_{0}\right)^{2}+\frac{\hbar}{m} t+\frac{\left(\Delta p_{0}\right)^{2}}{m^{2}} \cdot t^{2}
$$

This solution has three parts. The linear term is from classical diffusion and Einstein's kinetic theory [18].

$$
\begin{gathered}
\frac{\mathrm{d}}{\mathrm{d} t} f=\frac{\hbar}{2 m} \frac{\mathrm{d}^{2}}{\mathrm{~d} x^{2}} f \\
D=\mu k_{B} T=\frac{\tau}{m} k_{B} T \\
(\Delta x)^{2}(t)_{\text {linear }}=\frac{\hbar}{m} t
\end{gathered}
$$

The constant and quadratic parts are from quantum diffusion which is solved (typically by) Fourier Analysis on the kinetic energy Hamiltonian.

$$
\begin{aligned}
H & =\frac{p^{2}}{2 m} \\
\frac{\mathrm{d}}{\mathrm{d} t} \psi & =\frac{\mathrm{i} \hbar}{2 m} \frac{\mathrm{d}^{2}}{\mathrm{~d} x^{2}} \psi \\
(\Delta x)^{2}(t)_{\text {constant \& quadratic }} & =\left(\Delta x_{0}\right)^{2}+\frac{\left(\Delta p_{0}\right)^{2}}{m^{2}} \cdot t^{2}
\end{aligned}
$$

\subsection{Resistive Force}

In Section 3 we assumed a quadratic potential energy term and derived a resistive spring force. Here we will derive the same force but from kinematic arguments. If 
we look at classical diffusion term and consider the value at $t=\tau$

$$
2 D \tau=\frac{\hbar}{m} \tau=\frac{\hbar^{2}}{2 m k_{B} T}
$$

Next rearrange the diffusion constant

$$
2 D t=2 \mu k_{B} T t=\frac{-2 v t}{F} k_{B} T
$$

Replacing $x=v t$ and equating $2 D \tau$ to $2 D t$ we have,

$$
F=\frac{-m x}{\tau^{2}}
$$

\subsection{Modified Langevin Equation}

With a particle no longer free we must re-solve for the variance using the Langevin equation. However contrary to the ordinary Langevin equation $[15,18]$ we will change the assumption that the noisy driving force is uncorrelated with the particle's location. As we just derived, the force is anti-correlated with the position $F=-m r / \tau^{2}$. The $1-D$ equations of motion become,

$$
m(\ddot{x})=-\frac{m}{\tau}(\dot{x})-\frac{m}{\tau^{2}} x
$$

This equation can be used to solve $\overline{x^{2}}$ if one assumes the virial theorem [9] where the average quadratic potential energy is equal to the average kinetic energy. The initial condition $x(\dot{x})(t=0)=\hbar / 2 m$ is also assumed ensuring the equation's boundary conditions obey Heisenberg's Uncertainty. With calculus and the chain rule, one has,

$$
\overline{x^{2}}=\frac{\hbar^{2}}{2 m k_{B} T}\left(1-\mathrm{e}^{-2 k_{B} T t / \hbar}\right)=2 D \tau\left(1-\mathrm{e}^{-t / \tau}\right)
$$

This version of the Langevin equation has the familiar $2 D \tau$ term; however, it represents a stationary process where the ordinary Langevin equation is non-stationary.

\subsection{Stationary Diffusion}

The quantum solution from the modified Langevin presented in Section 4.3 is very interesting for two reasons. First it has a finite asymptotic value, which is what we would expect for a quantum solution to a black hole. We would expect that a black hole has a finite width to it and the outward diffusive pressure is balanced by an inward gravitational pressure.

Secondly we notice the asymptotic variance of position is twice that of $\left(\Delta x_{0}\right)^{2}$. This represents the dark particle cooling. As the particle diffuses out to $2\left(\Delta x_{0}\right)^{2}$ the temperature cools to $T / 2$ and the total energy in the oscillator goes from $3 k_{B} T$ to $3 k_{B} T / 2$. This is also where the particle gets the energy needed to radiate. The energy transfers over to the radiation field when the dark particle's wave function collapses as the photon is created. After the photon releases, the dark particle begins to diffuse again now at temperature of $T / 2$.

To remain consistent with our analysis above, if the photon is not collected by a neutral hydrogen atom before the next cycle of the harmonic oscillator begins, the photon will be re-absorbed, the dark particles' position will become fuzzy again and it will regain its energy and maintain its original temperature $T$.

\section{Quantum Solution (Discrete Space-Time)}

\subsection{Discrete Space Time}

Adding credibility to the modified Langevin equation, I simulate the outcome. Discrete space-time has been around for a while [19] and is becoming even more important [20]. To derive the correct model and the correct parameters for the model we will start with what we know.

\subsection{Standard Bernoulli Process}

The standard Bernoulli process is thoroughly reviewed by Reif [17] and Chandrasekhar [21]. In the standard Bernoulli process a particle steps to the right or steps to the left a distance $\delta x$ with probability $\beta, 1-\beta$ respectively at every time step $\delta t$. To derive the spatial step size, $\delta x$, we look at the variance as a function of the number of steps, $K$, and compare it to the continuous solution. Assuming $\beta=1 / 2$,

$$
(\Delta x)_{K}^{2}=\delta x^{2} K
$$

From Dirac's solution to the eigenvalue of the velocity [22] of a particle we know, $\delta x=c \delta t$, and since $t=K \delta t$, we have,

$$
(\Delta x)_{K}^{2}=\delta x \cdot c \cdot t
$$

A similar uncorrelated process in the momentum space gives us

$$
(\Delta p)_{K}^{2}=\delta p^{2} K
$$

From the virial theorem [9] we see, $\delta p=m c / K$, giving us the discrete linear solution

$$
(\Delta x)^{2}(t)=(\Delta x)_{K}^{2}+\frac{(\Delta p)_{K}^{2}}{m^{2}} t^{2}=2 \delta x \cdot c \cdot t
$$

From the continuous linear solution $(\Delta x)^{2}(t)=\hbar t / m$ one deduces,

$$
\delta x=\frac{\hbar}{2 m c}
$$




\subsection{Bernoulli Process with Resistive Force}

We can now proceed to modify the standard Bernoulli processes to account for the resistive force we found in the continuous case. We will find this discrete solution is equal to the modified continuous Langevin equation.

To begin with, we need to match the continuous 1-D force, $F=-m x / \tau^{2}$ with the ensemble average force experienced by the discrete case. The ensemble average force felt in the discrete case is the probability that the particle experiences a positive change in momentum times the impacted force $\delta p / \delta t$ plus the probability the particle experiences a negative change in momentum times $-\delta p / \delta t$. We can solve for the probability $\beta$ as a function of $x$ by examining the ensemble average discrete force on the particle felt at the location $x$ during time $\delta t$.

$$
F(x)=\beta(x) \frac{\delta p}{\delta t}+(1-\beta(x)) \frac{-\delta p}{\delta t}
$$

Solving for $\beta(x)$ we have,

$$
\beta(x)=\frac{1}{2}\left(1-\frac{m x \cdot \delta t}{\delta p \cdot \tau^{2}}\right)
$$

We can reduce this further since we know $\delta p$ and $\delta t$. Since we are dealing with the harmonic oscillator the only energy transition can be in multiples of the quantized energy of the oscillator, $\omega_{0}=2 k_{B} T$. Thus a change in momentum $\delta p$ must be equal to $2 k_{B} T / c$; $\delta t$ can also be replaced by $\hbar / 2 m c^{2}$ as described above.

$$
\beta(x)=\frac{1}{2}\left(1-\frac{x \cdot k_{B} T}{\hbar c}\right)
$$

The best way to show how this works is through a model where we show the variance of the position is equal to that of the modified continuous Langevin equation.

\subsection{Computer Model}

The following Matlab code shows the discrete model gives the same solution as the continuous modified Langevin. See Figures $\mathbf{4}$ and $\mathbf{5}$.

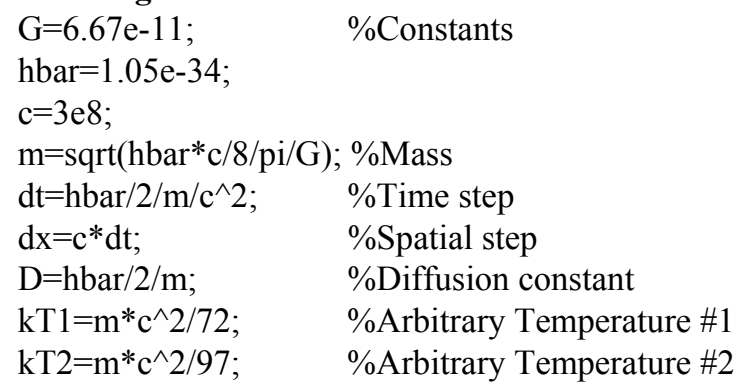

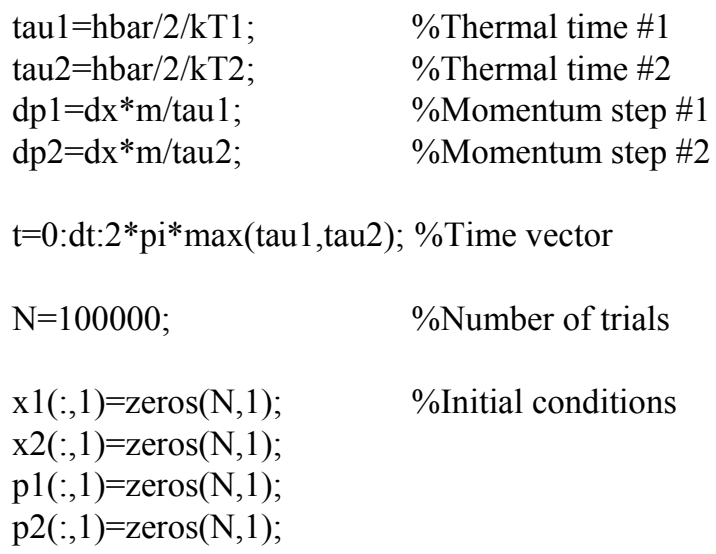

$\%$ Sample the probability and step $\mathrm{x} 1(:, \mathrm{k}+1)=\mathrm{x} 1(:, \mathrm{k})+\mathrm{dx} *(2 *$ floor $(\operatorname{rand}(\mathrm{N}, 1)+\mathrm{Bx} 1)-1)$; $\mathrm{x} 2(:, \mathrm{k}+1)=\mathrm{x} 2(:, \mathrm{k})+\mathrm{dx} *(2 *$ floor $(\operatorname{rand}(\mathrm{N}, 1)+\mathrm{Bx} 2)-1)$; $\mathrm{p} 1(:, \mathrm{k}+1)=\mathrm{p} 1(:, \mathrm{k})+\mathrm{dp} 1 *(2 *$ floor $(\operatorname{rand}(\mathrm{N}, 1)+\mathrm{Bp} 1)-1)$; $\mathrm{p} 2(:, \mathrm{k}+1)=\mathrm{p} 2(:, \mathrm{k})+\mathrm{dp} 2 *(2 *$ floor $(\operatorname{rand}(\mathrm{N}, 1)+\mathrm{Bp} 2)-1)$;

end

$\%$ Update position to include momentum contribution $\mathrm{x} 1=\mathrm{x} 1+\mathrm{p} 1 * \operatorname{tau} 1 / \mathrm{m}$; $\mathrm{x} 2=\mathrm{x} 2+\mathrm{p} 2 * \operatorname{tau} 2 / \mathrm{m}$

figure(4)

$\%$ Calculate variance from discrete model

$\mathrm{xvar} 1=\operatorname{mean}(\mathrm{x} 1 . * \mathrm{x} 1)-\operatorname{mean}(\mathrm{x} 1) .^{\wedge} 2$;

$\mathrm{xvar} 2=\operatorname{mean}\left(\mathrm{x} 2 .{ }^{*} \mathrm{x} 2\right)-\operatorname{mean}(\mathrm{x} 2) .^{\wedge} 2$;

$\%$ Calculate variance from Langevin langevin $1=2 * \mathrm{D} * \operatorname{tau} 1 *(1-\exp (-\mathrm{t} / \operatorname{tau} 1))$;

langevin $2=2 * \mathrm{D} * \operatorname{tau} 2 *(1-\exp (-\mathrm{t} / \mathrm{tau} 2))$;

$\operatorname{plot}(\mathrm{t}, \mathrm{xvar} 1)$

plot(t,langevin $\left.1, \mathrm{r}^{\prime}\right)$

plot(t,xvar2,'g')

plot(t,langevin2,'k')

figure(5)

sigma $=\operatorname{sqrt}(2 * \mathrm{D} * \operatorname{tau} 1) ; \quad \%$ Asymptotic variance $\mathrm{x}=-4 *$ sigma: $\mathrm{dx} / 10: 4 *$ sigma; \%Position vector

$\mathrm{P}=$ hist $(\mathrm{x} 1($, length $(\mathrm{t})), \mathrm{x}) / \mathrm{N} ; \%$ Calculate PMF

$\%$ Calculate PDF

$\mathrm{p}=1 / \operatorname{sqrt}\left(2 * \mathrm{pi}^{*} \operatorname{sigma}^{\wedge} 2\right)^{*} \exp \left(-\mathrm{x} .^{\wedge} 2 / 2 /\left(\operatorname{sigma}^{\wedge} 2\right)\right)^{*} \mathrm{dx}$;

$\operatorname{plot}(\mathrm{x}, \mathrm{P})$

$\operatorname{plot}\left(\mathrm{x}, \mathrm{p}, \mathrm{r}^{\prime}\right)$ 


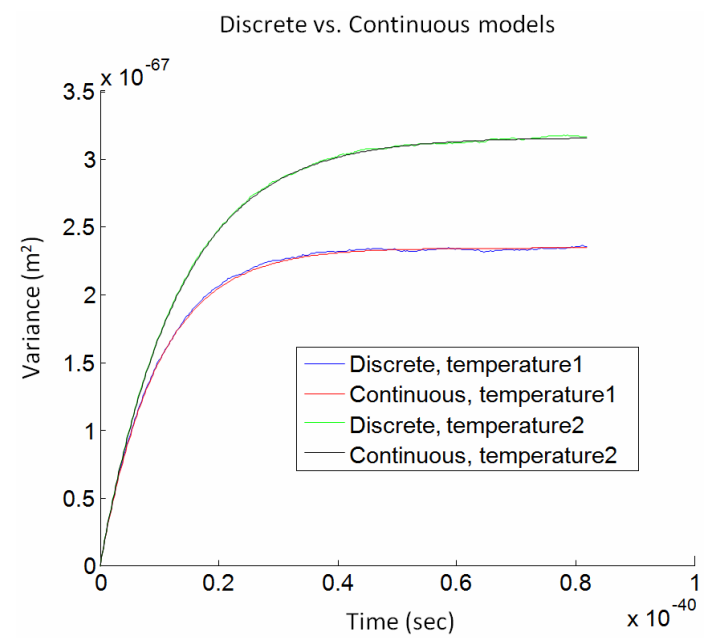

Figure 4. The variance in position of a dark particle; comparison between the discrete model and the exact continuous solution at two different temperatures.

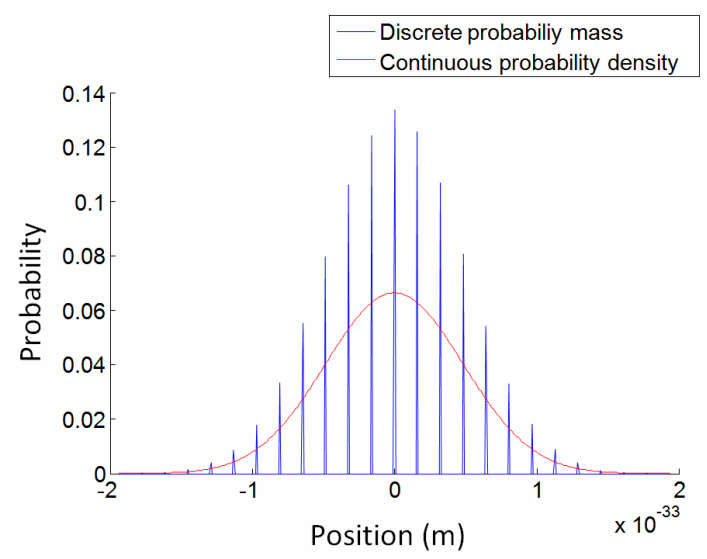

Figure 5. Probability distributions for one temperature; a comparison between discrete model and continuous theory.

\section{Friedmann's Equations Solutions}

We now show that by combining the energy density with three different equations of state, $w=-1,-1 / 3, \& 1 / 3$ we arrive at the same solution as what was derived in both the continuous and discrete quantum solutions. The solutions to Friedmann's equation with the densities standing alone correspond to the solutions to the linear and quadratic time terms of the variance when the particle is free. We need to assume the particles comes as pairs such that we can define a general relativistic length scale $L$ and a quantum mechanical length scale $\ell$.

\subsection{Length Scales}

We define $L$ as twice the light time $\tau$, the maximum distance two particles can traverse in time $\tau$.

$$
L \equiv 2 \tau c=\frac{\hbar c}{k_{B} T}
$$

We define $\ell$ as the variance between the two particles. If the two particles have independent wave functions we have

$$
\ell \equiv \sqrt{2} \cdot \Delta x
$$

Using these two definitions we will show that under three different equations of state $L$ (the solution to Friedmann's equation) will be equal to $\ell$ (the variance of quantum diffusion).

\subsection{Equation of State, $w=1 / 3$}

First for the equation of state $w=1 / 3$, we have the energy in the 3-D oscillator

$$
\hbar \omega=\frac{m}{2 \tau^{2}}\left(x^{2}+y^{2}+z^{2}\right)+\frac{1}{2 m}\left(p_{x}^{2}+p_{y}^{2}+p_{y}^{2}\right)
$$

We begin with the probability distribution on $\omega \sim p(\omega, T) d \omega$ from Section 3.2

$$
\omega \sim p(\omega, T) d \omega=\frac{\hbar^{3} \omega^{2}}{2\left(k_{B} T\right)^{3}} \mathrm{e}^{\frac{-\hbar \omega}{k_{B} T}} d \omega
$$

Since we are no longer talking about photons/bosons like above but are talking about fermions we don't have to account for multiple particles. The average energy of this distribution is the three dimensional ground state energy of the harmonic oscillator, $3 k_{B} T$.

If we consider a volume $V=L^{3}$ the energy density is

$$
\rho_{w=1 / 3}(L)=\frac{3 k_{B} T}{c^{2}(L)^{3}}=\frac{3 \hbar}{c(L)^{4}}
$$

The Friedmann equation when the density is dominated by this equation of state, $w=1 / 3$ becomes,

$$
\left(\frac{(\dot{L})}{L}\right)^{2}=\frac{8 \pi G}{3} \rho_{w=1 / 3}(L)=\frac{8 \pi G \hbar}{c(L)^{4}}
$$

Solving for $L(t)$ we see it is equal to linear diffusive term from Section 4.1.

$$
L_{w=1 / 3}^{2}=\left(\frac{32 \pi G \hbar}{c}\right)^{1 / 2} t=\frac{2 \hbar}{m} t=\ell_{\text {linear }}^{2}
$$

\subsection{Equation of State, $w=-1 / 3$}

In deriving the density and solution for this equation of state we turn to a derivation of Friedmann's equation [1]. We will start by deriving the gravitational explanation of the resistive spring force. Equating the average gravitational potential energy to $3 k_{B} T / 2$ for 3 dimensions gives,

$$
\overline{P E_{\text {gravity }}}=\frac{\overline{-G M m}}{r}=\frac{3 k_{B} T}{2}
$$


When $M=4 \pi r^{3} \rho / 3$

$$
\frac{\overline{-G M m}}{r}=\frac{-4 \pi G \rho m \overline{r^{2}}}{3}
$$

Due to symmetry we can re-write $\overline{r^{2}}$ as $3\left(\Delta x_{0}\right)^{2}=3 \hbar^{2} / 4 m k_{B} T$ [9] to arrive at,

$$
\frac{-8 \pi G \rho}{3}=\frac{\left(2 k_{B} T\right)^{2}}{\hbar^{2}}
$$

Plugging this back into the relationship between potential energy and force [9] and with time constant $\tau=\hbar / 2 k_{B} T$ we have,

$$
\begin{aligned}
F_{r} & =\frac{-\mathrm{d}}{\mathrm{d} r} P E=\frac{-\mathrm{d}}{\mathrm{d} r}\left(\frac{-G M m}{r}\right)=\frac{-\mathrm{d}}{\mathrm{d} r}\left(\frac{-4 \pi G \rho m r^{2}}{3}\right) \\
& =\frac{8 \pi G \rho m r}{3}=\frac{-4 m\left(k_{B} T\right)^{2}}{\hbar^{2}} r=\frac{-m r}{\tau^{2}}
\end{aligned}
$$

When a particle moves within the space curved by the black hole, a resistive spring force is in play. Here we see a gravitational explanation for the spring force.

Going back to solve for the density we have,

$$
\frac{-4 \pi G \rho m \overline{r^{2}}}{3}=\frac{3 k_{B} T_{0}}{2}
$$

Where $T_{0}$ is constant. With $\overline{r^{2}}=3\left(\Delta x_{0}\right)^{2}=3 \ell^{2} / 2$ and $m$ the reduced Planck mass, the density is

$$
\rho_{w=-1 / 3}(\ell)=\frac{-6 m k_{B} T_{0}}{\hbar c(\ell)^{2}}
$$

Friedmann's equation and its solution show $L_{w=-1 / 3}$ is equal to the quadratic diffusive term from Section 4.1.

$$
\begin{gathered}
\left(\frac{(\dot{L})}{L}\right)^{2}=\frac{8 \pi G}{3} \rho_{w=-1 / 3}(L)=\frac{-2 k_{B} T_{0}}{m(L)^{2}} \\
L_{w=-1 / 3}^{2}=\left(\frac{-2 k_{B} T}{m}\right) t^{2}=-\ell_{\text {quadratic }}^{2}
\end{gathered}
$$

Notice the solution of $L_{w=-1 / 3}$ is imaginary because of the positive curvature associated with this equation of state [1]. Yet we still have $|L|=|\ell|$. In the next two paragraphs we derive the holistic energy density, $\rho_{\text {dark particle }}$ which is always positive and thus $L_{\text {dark particle }}$ is real.

The last term we need is a constant energy density, $w=-1$. To solve for the constant density, we insert $L_{0}=\hbar / \sqrt{m k_{B} T_{0}}$ (which we show is the asymptotic value of the solution) into the density of the oscillator.

$$
\rho_{w=-1}=\rho_{w=1 / 3}\left(L=L_{0}\right)=\frac{3 \hbar}{c\left(L_{0}\right)^{4}}=\frac{3 m^{2}\left(k_{B} T_{0}\right)^{2}}{c \hbar^{3}}
$$

The solution to Friedmann's equation with this density is exponentially increasing, however if we add this density term to our other two densities we see the solution is equal to the solution of the Langevin equation.

$$
\begin{gathered}
\rho_{\text {dark partilce }}=\rho_{w=-1}+\rho_{w=-1 / 3}+\rho_{w=1 / 3} \\
\rho_{D P}(L)=\frac{3}{\hbar c}\left(\frac{\hbar^{2}}{(L(t))^{4}}-\frac{2 m k_{B} T_{0}}{(L(t))^{2}}+\frac{m^{2}\left(k_{B} T_{0}\right)^{2}}{\hbar^{2}}\right)
\end{gathered}
$$

By applying calculus, the solution to Friedmann's equation with this density is

$$
\begin{gathered}
\left(\frac{(\dot{L})}{L}\right)^{2}=\frac{8 \pi G}{3} \rho_{D P}(L)=\left(\frac{\hbar}{m L^{2}}-\frac{k_{B} T_{0}}{\hbar}\right)^{2} \\
L_{D P}^{2}=\frac{\hbar^{2}}{m k_{B} T_{0}}\left(1-\mathrm{e}^{-2 k_{B} T_{0} t / \hbar}\right)
\end{gathered}
$$

With $k_{B} T_{0}=\hbar /\left(2 \tau_{0}\right)$ and the $D=\hbar /(2 m)$, this is re-written, showing $L_{\mathrm{DP}}$ equal to the stationary Langevin equation from Section 4.3.

$$
L_{D P}^{2}=4 D \tau_{0}\left(1-\mathrm{e}^{-t / \tau_{0}}\right)=\ell_{\text {Langevin }}^{2}
$$

We see the solutions to Friedmann's equation and the equations of quantum diffusion behave in the same way. It is interesting to note that the density vanishes at the asymptotic value $\rho_{D P}\left(L_{0}\right)=0$ so we don't have to worry about this fermionic density contributing to the cosmological constant.

\section{Discussion}

A similarity one might find with other current work would be Primordial Black Hole Remnants (PBHR). Chen uses PBHRs nicely to explain dark matter [23]. Interpreted here the density of state is determined by how the density scales as a function of the length scale. We have suggested that when the dark particles cannot couple to ordinary matter the temperature and thus density is frozen, implying a density of state of $w=-1$ and explaining dark energy. However if the dark particles are near neutral hydrogen atoms (et al. near galaxies), allowing them to couple and release heat, the density of state could be positive and the acceleration equation would be positive, more in line with dark matter [1].

A test of the hypothesis that if neutral hydrogen atoms are near, dark particles act like more like dark matter than dark energy would be to look for cosmological observations where we observe either bountiful or scanty amount of neutral hydrogen. Perhaps Virgo21 (where we observe neutral hydrogen atoms and possibly a high density of dark matter) [24] or global clusters (where any neutral atoms would be ionized and almost no dark mat- 
ter) [25] could serve as a first validation.

A further validation of this hypothesis might be experimentally possible on Earth. A team led by Perl [26] has suggested two identical side-by-side atom interferometers could reveal a dark energy density by measuring the time it takes for atoms to fall through gravity, respectively between the two interferometers. One could build on this approach and artificially alter the density of the dark particles by surrounding each interferometer in a bath of neutral hydrogen atoms (or other suitable sink that is not dangerous at high temperatures). One bath could be kept at a low temperature and the other at a high temperature. If the bath was large enough to allow the neutral hydrogen and the dark particles to couple and exchange heat before the dark energy reference frame moves past the interferometers [26], the density of dark particles that interacts with the falling atoms would be different between the hot and the cold interferometers and the difference would be measured.

Questions still remain, like the exact mechanism for how the dark particle exchanges heat, and more analysis is needed for dark particles fully to answer the questions of dark energy [2,5], or for that matter other open questions like dark matter [27]. Yet this initial brief report is intended to set the physical parameters and give guidance for how the forces of gravity and quantum mechanics work together and have complementary solutions in a simple straightforward way.

While it is not possible to create a particle with the reduced Planck mass artificially, it would explain why prior experiments have been unable to locate the missing energy.

As a note, the dark particle was built out of research in Finite Difference Time Domain (FDTD) modeling of diffusive motion [19]. By noticing a connection between Bernoulli's process [17,21] and black-body radiation [17] it was possible to derive the continuous version of the theory. Application to Friedmann's equation followed a need to explain the resistive spring force that keeps the particle stationary. When the theory suggested a density of black-body radiation was hidden (because the cross section of the dark particle is on the order of the Planck length) the tie to dark energy was made.

Mountain View, CA, March 2011.

\section{Acknowledgements}

JLH would like to thanks his family who encouraged him to stick with his work. JLH also would like to call out those around the world who helped, specifically in the Electrical Engineering and Physics departments at Princeton and Stanford where the foundation of this research was built.

\section{REFERENCES}

[1] A. Liddle, “An Introduction to Modern Cosmology," 2nd Edition, John Wiley \& Sons, Ltd., W. Sussex, 2003.

[2] P. Peebles and B. Ratra, Reviews of Modern Physics, Vol. 75, 2003, pp. 559-606. doi:10.1103/RevModPhys. 75.559

[3] D. Larson, J. Dunkley, et al., "Seven-Year Wilkinson Microwave Anisotropy Probe (WMAP) Observations: Power Spectra and WMAP-Derived Parameters," 2010. arXiv: $1001.4635 \mathrm{v} 2$

[4] S. E. Rugh and H. Zinkernagel, Studies in History and Philosophy of Modern Physics, Vol. 33, 2002, pp. 663705. doi:10.1016/S1355-2198(02)00033-3

[5] B. Greene, "The Fabric of the Cosmos," Vintage Books, New York, 2004.

[6] L. Papantonopoulos, "Lecture Notes in Physics 720 The Invisible Universe: Dark Matter and Dark Energy," Springer, Berlin, 2007. doi:10.1007/978-3-540-71013-4

[7] L. Amendola and S. Tsujikawa, "Dark Energy: Theory and Observations," Cambridge University Press, Cambridge, 2010. doi:10.1017/CBO9780511750823

[8] S. W. Hawking, Communications in Mathematical Physics, Vol. 43, 1975, pp. 199-220. doi:10.1007/BF02345020

[9] Feynman, "Lectures on Physics," Addison-Wesley Publishing, Reading, 1965.

[10] C. W. Gardiner and P. Zoller, "Quantum Noise," Springer, Berlin, 2004.

[11] C. Barcelo, S. Liberati, S. Sonego and M. Visser, Physical Review D, 83, 2011, Article ID: 041501. doi:10.1103/PhysRevD.83.041501

[12] S. A. Hayward, Classical and Quantum Gravity, Vol. 15, 1998, p. 3147. doi:10.1088/0264-9381/15/10/017

[13] R. Barkana and A. Loeb, Physics Reports, Vol. 349, 2001, pp. 125-238. doi:10.1016/S0370-1573(01)00019-9

[14] A. R. Liddle and D. H. Lyth, "Cosmological Inflation and Large-Scale Structure," Cambridge University Press, Cambridge, 2000. doi:10.1017/CBO9781139175180

[15] D. F. Walls and G. J. Milburn, "Quantum Optics," Springer, Berlin, 1994.

[16] J. Pan, "Email Correspondence," Fudan University, Shanghai, 2007.

[17] Reif, "Fundamentals of Statistical and Thermal Physics," McGraw Hill, Boston, 1965.

[18] R. Kubo, Reports on Progress in Physics, Vol. 29, 1966, p. 255. doi:10.1088/0034-4885/29/1/306

[19] Forsythe \& Wasow, "Finite-Difference Methods for Partial Differential Equations," John Wiley \& Sons, Inc., New York, 1960.

[20] M. Mecklenburg and B. C. Regan, Physical Review Letters, Vol. 106, 2011, Article ID: 116803. doi:10.1103/PhysRevLett.106.116803

[21] Chandrasakhar, Reviews of Modern Physics, Vol. 15, 1943.

[22] P. A. M. Dirac, "The Principles of Quantum Mechanics," 4th Edition, Oxford University Press, Oxford, 1958, p. 262. 
[23] P. Chen, "Generalized Uncertainty Principle and Dark Matter," Quantum Aspects of Beam Physics, World Scientific Publishing, Singapore, 2004, p. 315.

[24] R. Minchin, et al., Astrophysics Journal, Vol. 670, 2007, pp. 1056-1064. doi:10.1086/520620

[25] S. Mashchenko and A. Sills, Astrophysical Journal, Vol. 619, 2005, pp. 243-257. doi:10.1086/426132
[26] R. J. Adler, H. Mueller and M. L. Perl, "A Terrestrial Search for Dark Contents of the Vacuum, Such as Dark Energy, Using Atom Interferometry," 2011. arXiv:1101.5626v1

[27] Bertone \& Silk, "Particle Dark Matter: Observations, Models, and Searches," Cambridge University Press, Cambridge, 2010. 